\title{
Antidepressant prescribing in general practice - how long can this go on?
}

\author{
Michael P. KeRR, Senior House Officer in Psychiatry, Whitchurch Hospital, \\ Cardiff CF4 7XB
}

Present guidelines for the prescribing of antidepressant medication, with particular reference to the duration of treatment, are unclear (BNF, 1989). Evidence suggests that continuation of treatment for at least six months is valuable in the prevention of relapse of the acute illness (Mindham et al, 1975). Other workers have demonstrated a prophylactic effect similar to lithium over a three year period in patients with unipolar depression. There is, however, a paucity of information about the benefits (or lack of benefits) of longer term maintenance treatment. This study focuses on a population of a type which has been previously studied elsewhere - of patients in general practice receiving long-term (greater than one year) antidepressant medication. The implications of this to the psychiatrist and general practice-psychiatry liaison are discussed.

\section{The study}

The study was based in a large group practice consisting of nine partners covering a population of approximately 20,000 patients. The area covered was a combination of both rural and urban housing. The practice computer was used to identify those patients who had repeat prescriptions of antidepressant medication (excluding monoamine-oxidase inhibitors) available to them. The patients' notes were retrieved and a retrospective analysis carried out.

\section{Findings}

Age-sex distribution

A total of 147 patients were identified. Of them 107 (72\%) were women whose age range was from 32-89 years. Nineteen were under 55 while 41 were over 70 . Forty patients $(28 \%)$ were male with an age range from 32-89 years.

\section{Contact with psychiatric services}

Eighty-six (58\%) had never seen a psychiatrist previously, whereas $61(42 \%)$ had been psychiatric in-patients or received out-patient care.

\section{Drug dosage}

Of the 147 patients, there was adequate information on dosages for 145 . Of these, 126 were receiving tricyclic antidepressants (amitriptyline, clomipramine, dothepine, desimipramine, imipramine, lofepramine and trimipiramine). The dosage prescribed were generally low; 51 patients received 50 mgms or less daily; 40 received $70-75 \mathrm{mgms}$ daily and 26 were receiving 100 mgms or more.

\section{Follow-up}

Follow-up was measured by consultations with the general practitioner in the preceding year. It was not possible to discover whether these consultations had a psychiatric content but they at least confirmed some doctor-patient contact. When the rates of consultations were compared with the national averages, they were found to be higher in the study population in all the age-sex ranges; in particular males aged 16-44 attended on average eight times whereas the national average was two.

\section{Duration of antidepressant treatment}

This is summarised in Fig. 1. The figure shows a marked reduction in prescribing from 3-5 to 6-10 years. There is no obvious reason for this, though it may reflect prescribing habits or the natural progression of the illness.

\section{Comment}

The tendency to prescribe comparatively low dosages of antidepressants in general practice has been observed previously (Catalan et al, 1988; Johnson, 1974; Tyrer, 1978). It has been suggested that such prescribing is inappropriate and sub-optimal and that follow-up is poor (Johnson, 1974). However, although this study confirmed the generally low doses, the follow-up as judged by the crude measure of consultation rates appeared acceptable.

Obviously the use of consultation rates as a measure of follow-up is flawed. It may be that the patient's prescriptions were not assessed at all, or 


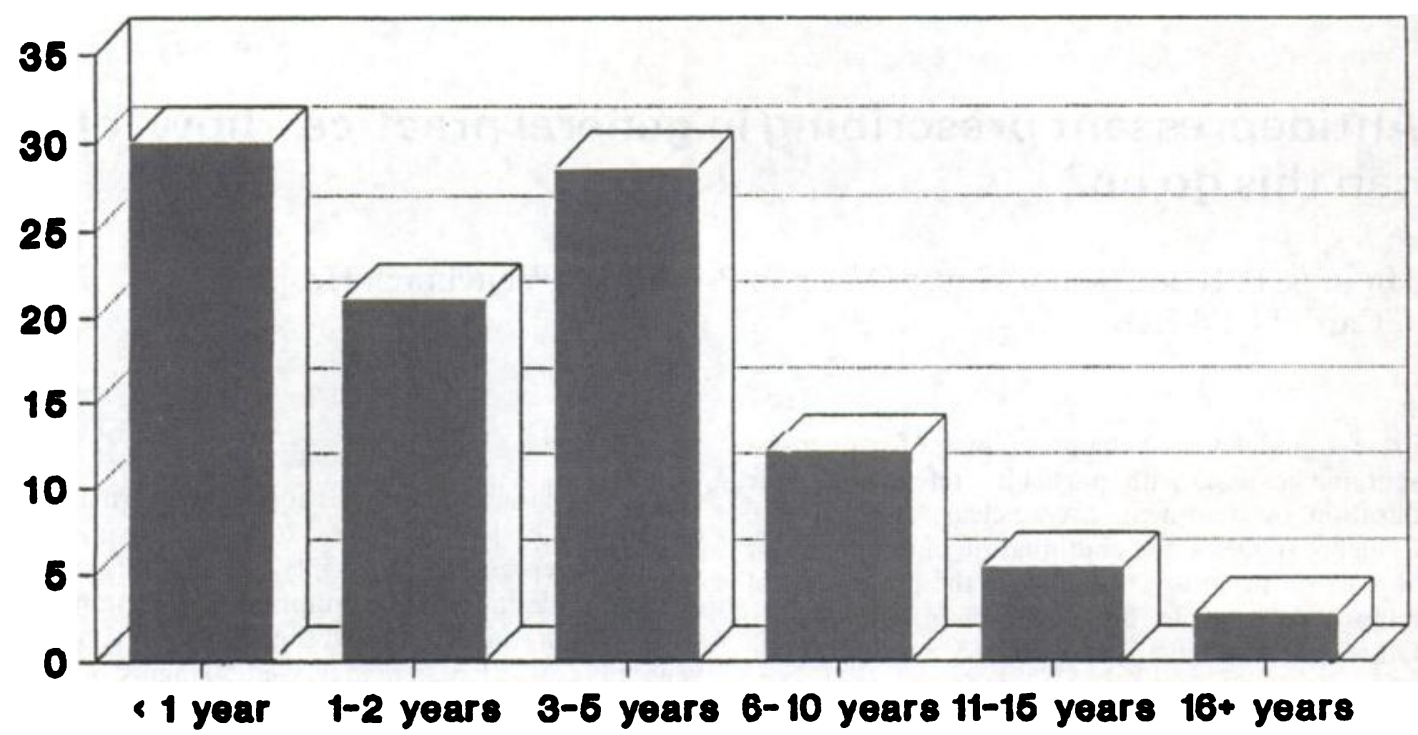

percent of patients

FIG. 1. Duration of prescription of antidepressants

that the consultation was patient-orientated. However, in a retrospective study consultation rates at least confirm a point of contact between the GP and his patient.

The identification of this group of patients receiving long-term prescription of antidepressant medication as shown here and in other studies (Catalan et al, 1988) is of particular importance for although research has shown the advantage of continuation of antidepressant medication in the short term (Mindham et al, 1975) the evidence for the need for long-term prescription is less clear.

The characteristics of this group are interesting. They are more likely to be older and to have had previous psychotropic medication; they also have a high incidence of previous psychiatric contact, deliberate self harm and social disturbance (Catalan et al, 1983). As such they are a group who would appear to be at particular risk from the toxicity of antidepressant medication, especially in overdosage. They are therefore, as a group, of particular interest to psychiatry. This interest should be focused on two main issues. Firstly, to discover why these people receive such therapy? Secondly, how can we help the GPs with this group of patients? One possibility is that these patients all suffer from unipolar affective disorder and are gaining benefit from prophylactic antidepressant therapy. This may be the case, but we have no evidence to suggest it and such a homo- geneous group is unlikely. Certainly in this study the information recorded in the notes was insufficient to apply recognised diagnostic criteria and therefore no attempt was made to assess the diagnosis. This is a handicap of retrospective work. As to the actual diagnosis, I am sure a further analysis (maybe using PSE as by Catalan et al) would reveal a heterogeneous group, possibly containing personality disorders and anxiety neurosis. These disorders are sometimes difficult to differentiate from depression; patients are often chronic repeated attenders and may be gaining benefit from the anxiolytic properties of the antidepressants. As already mentioned, there are certain sociological characteristics of these people (previous deliberate self-harm, social disturbance, and high incidence of previous psychiatric contact) which may influence their need for long-term therapy. The answer is surely more complex. It is likely to be a combination of the prescribing habits of the GP and personal characteristics of the patient influencing the doctor's ability to discontinue antidepressant therapy. The prescribing policy of the local psychiatric services may well also influence this.

It is the responsibility of the psychiatrist to help the GP with this problem. Ideally help should be based on the evaluation of the efficacy of such therapy. Unfortunately most psychiatric research has been based on medium term (6-9 month) maintenance-indeed this is often the length of follow-up 
in clinic before the patient is returned to the care of the GP, so longer prospective trials may help. It will also be important to establish the diagnosis of the patients, for with knowledge of the likely diagnostic categories and outcomes some rational scheme can surely be devised - perhaps in the form of guidelines on maintenance prescription of antidepressants in general practice-which may well help GPs to know just "how long" they can go on.

\section{Acknowledgement}

I am very grateful for the advice given to me by Professor P. McGuffin in writing of this paper.

\section{References}

British National Formulary, Number 17 (March 1989).

Catalan, J., Gath, Dh., Bond, A., Edmonds, G., Martin, P. \& ENNIS, J. (1988) General practice patients on longterm psychotropic drugs: A controlled investigation. British Journal of Psychiatry, 152, 399-406.

JoHnson, D. (1974) A study of the use of antidepressant medication in general practice. British Journal of Psychiatry, 125, 186-192.

Mindham, R. H. S., Bagshaw, A., Howard, C. \& SHEPHERD, M. (1975) An evaluation of continuation therapy with tricyclic antidepressants in depressive illness. Psychological Medicine, 3, 5-17.

TYRER, P. J. (1978) Drug treatment of psychiatric patients in general practice. British Medical Journal, ii, 1008-1010.

\title{
Medical audit quality of note-keeping
}

\author{
Aristos Markantonakis, Senior Registrar; and I. K. WeIR, Consultant in Child \\ Psychiatry, The Institute of Family Psychiatry, 23 Henley Road, Ipswich, \\ Suffolk IP1 3TF
}

Recently there has been an increasing awareness of the need for medical audit. Medical audit in the health service is not a new thing across the Atlantic. Many centres accept it as a normal part of health management. The findings of audit are made known to the individuals who have been audited with the aim that this would produce future improvement. Hence it is an effective way of increasing efficiency and highlighting inefficient and costly procedures. There is debate as to who should be doing the auditing. General practitioners have been suggested as being in the ideal position to be able to audit specialist services such as psychiatric services (Ferguson, 1990). GPs can be approached by questionnaire to gain their views as to the level of satisfaction on things such as communication, availability and usefulness of referral to a local specialist service (Markantonakis \& Mathai, 1990).

The quality of note-keeping by professionals in a department is very important. This importance is highlighted by the increasing amount of medico-legal work that is demanded from psychiatrists by courts. We are constantly reminded that medical notes can be referred to in court and that their accuracy can be of paramount importance. Child psychiatry has seen an increase in medico-legal work and an explosion in awareness and referral of cases of child abuse, physi- cal, emotional, and sexual. Who will be responsible for checking the quality of note-keeping? From our experience, constant reminders by the doctors to other professionals working on the multidisciplinary team is not sufficient to increase the quality of note keeping. Therefore we felt there was a need for a formal method of quality check.

\section{The questionnaire}

We devised a questionnaire asking seven questions which require a Yes or No answer. The questionnaire is easy to fill in and takes about five minutes to complete. The questions are designed to draw the attention of the note-keeper to the following points:

(1) The importance of providing a written entry in the notes after the first contact interview with a family or patient.

(2) The legibility of this entry.

(3) The importance of providing a 'formulation' in the case notes. The formulation should include a diagnosis or a clear description of the problem (everybody knows that ICD-10 and DSM-III$\mathbf{R}$ diagnoses are not easily provided by nondoctors) and secondly, the formulation should include a clear plan of management for the patient. 